\title{
PROGRESS TOWARD HIGH ENERGY ELECTRON COOLING
}

\author{
S. Nagaitsev ${ }^{\dagger}$, FNAL $^{*}$, Batavia, IL 60510, USA
}

\begin{abstract}
All electron cooling systems in operation to date can be classified as low energy systems. The electron beam kinetic energy in such a system is limited to about $0.6-1$ $\mathrm{MeV}$ by the use of a conventional commercial CockcroftWalton high-voltage power supply. This, in turn, bounds the maximum ion kinetic energy, accessible for cooling with today's standard technology, to about $2 \mathrm{GeV} /$ nucleon (about a factor of 2 - 3 times higher than the electron systems in operation today). Electron cooling systems with kinetic energies above $1 \mathrm{MeV}$ could provide economically justifiable improvements in the performance of many existing and proposed accelerator complexes, such as RHIC, Tevatron and HERA. This paper reviews the status of the development of the technology needed for high energy electron cooling.
\end{abstract}

\section{INTRODUCTION}

According to the well-proven concept of electron cooling [1], the electron beam, needed to cool the ions/protons, has to co-propagate with the ion/proton beam in a straight section of the ring free of ion-beam focusing magnets. The velocities of two co-propagating beams have to precisely coincide, thus requiring, for example, an electron beam energy of about $25 \mathrm{MeV}$ for a proton energy of $50 \mathrm{GeV}$. The portion of the ring dedicated to the electron cooling system has to be as long as possible (generally, cooling rate is proportional to this length) but typically comprises no more than a few per cent of the ring's circumference.

I define a "high energy" as the energy at which the conventional "low-energy" electron cooling technologies and techniques for the production and transport of the electron beams become difficult or cumbersome. These include:

- The power supply (or electron beam energy source) technology;

- The technique to transport the electron beam in a continuous magnetic field from the cathode to the cooling section to the collector;

- A relatively short cooling section (typically $2 \mathrm{~m}$ ), which comprises 2 to 5 per cent of the ring circumference.

Cooling beam at high energies has an apparent advantage over cooling at low energies, prior to acceleration. First, the space charge effects, which at low energies limit the achievable emittances, are significantly reduced at relativistic energies, thus allowing for deeper cooling. Second, electron cooling at high energies can

\footnotetext{
${ }^{\dagger}$ nsergei@fnal.gov

*Operated by Universities Research Association, Inc., under Contract No. DE-AC02-76CH03000 with the U.S. Department of Energy
}

help accumulation of rare beams (such as antiprotons) that are produced only at high energies. Third, electron cooling at the top energy of a collider or a storage ring with an internal target can significantly increase the luminosity by counteracting various diffusion effects [10].

Following the above definition, the transition from the low-energy to the high-energy probably lies above electron kinetic energies of about $1 \mathrm{MeV}$. Even though the use of low energy electrons $(<300 \mathrm{keV})$ for cooling is a well-developed technology, the electron energy of 1 $\mathrm{MeV}$ or higher coupled with a relatively high required average beam current (about $100 \mathrm{~mA}$ or greater) posses a significant technological hurdle.

For electron kinetic energies of up to about $5-8 \mathrm{MeV}$ (ion energies $10-15 \mathrm{GeV} /$ nucleon) technically it is quite possible to use a conventional dc electrostatic accelerator in a recirculation regime. Budker INP (Novosibirsk) has developed a technology for a $1 \mathrm{MeV}$ (kinetic), $1 \mathrm{~A} \mathrm{dc}$ recirculation system operating in a continuous magnetic field of $500 \mathrm{G}$ [2]. The high-voltage power supply was based on an industrial high-power electron accelerator, developed at INP. This system would require many modifications in order to be extended to a 5-8 MV range. Its development is not being currently pursued for energies higher than $1.5 \mathrm{MeV}$ [3]. A proposal to use a modified betatron to accelerate and then to store a highenergy electron beam for electron cooling is being currently investigated at JINR [4]. DESY is studying a possibility of electron cooling $18 \mathrm{GeV} / \mathrm{c}$ protons in Petra [5]. Fermilab is currently developing a $5 \mathrm{MeV}$ dc electron cooling system to cool $8.9 \mathrm{GeV} / \mathrm{c}$ antiprotons [6]. To date, this is the only fully funded $R \& D$ project that would qualify (if successful) as a high energy system. For higher energies (up to hundreds of MeV's) the most promising approach would appear to be the rf acceleration of bunched electron beams in an energy-recovering linac system [7]. Successful operation of such an accelerator has been recently demonstrated by a free-electron laser group at the Jefferson Lab [8]. An excellent review of various high-energy cooling approaches was presented in Ref. [9] and [10]. In this paper I will present R\&D issues that are being currently investigated at Fermilab as well as at other labs in order to achieve an operating high-energy electron cooling systems.

\section{ELECTRON BEAM MAGNETIZATION}

Electron cooling in a solenoidal magnetic field (with immersed gun cathode) is a traditional optical solution at low energies. In fact, there has never been a demonstration of electron cooling without the accompanying magnetic field. The presence of a 
longitudinal magnetic field is also considered beneficial at high energies for two reasons:

- the solenoidal magnetic field allows to combine strong focusing with the requirement (for efficient cooling) of low electron transverse temperature in the cooling interaction region;

- cooling rates with a "strongly" magnetized electron beam are ultimately determined by the electron longitudinal energy spread only, which can be made much smaller than the transverse one.

An electron beam is considered magnetized when its radius of transverse Larmor oscillations is much smaller than the beam radius. If the solenoidal field lines are perfectly parallel, these oscillations (among other conditions) can increase the duration of an electron-ion interaction thus increasing the friction force (assuming that there are at least several Larmor oscillations in the cooling section). Such a magnetization is considered "strong" as far as the cooling process is concerned. At high energies $(\gamma=10-100)$ this requires a continuos solenoidal field of $1-10 \mathrm{kG}$ and the cooling section of at least $20-30 \mathrm{~m}$. On the other hand, it is believed possible to preserve electron transverse temperatures at a low (thermal) level and to employ only weak magnetic field (but strong enough to provide focusing against electron beam space charge). Such a field can be about $100 \mathrm{G}$ or less. Both of these two field options are being currently considered for high energy electron cooling projects.

The most serious question is how to produce a long solenoid of required field quality and how to measure it. Generally speaking, the field quality (defined as the ratio of the transverse field component to the longitudinal one) requirement at high energies is more severe (by about a factor of $\gamma$ ) than at low energies.

\subsection{High field option (RHIC cooling system)}

The researchers at Budker INP and BNL, who proposed electron cooling of gold ions in RHIC, have encountered an interesting problem related to the ion recombination during the cooling process if the electron beam is cold. To suppress this recombination it is proposed to increase the electron temperature to $1000 \mathrm{eV}$ and rely on "strongly" magnetized cooling. The electron cooler and beam parameters under consideration are:

Beta-function in the cooling section: $\beta=60 \mathrm{~m}$

Ion beam normalized rms emittance: $\varepsilon_{n}=1 \mu \mathrm{m}$

Ion beam rms angular spread: $\quad \theta_{i}=1.3 \times 10^{-5} \mathrm{rad}$

Electron beam momentum: $\quad p c=50 \mathrm{MeV}$

Relativistic parameter: $\quad \gamma=100$

Electron beam transverse temperature: $T_{e}=1000 \mathrm{eV}$

Electron beam rms angular spread: $\quad \theta_{e}=6 \times 10^{-4} \mathrm{rad}$

Magnetic filed in the cooling section: $H_{0}=1 \mathrm{~T}$

Solenoid length: $\quad L=30 \mathrm{~m}$

Larmor radius (at full energy): $\quad \rho=p c / e H_{0} \approx 16.6 \mathrm{~cm}$

Larmor period: $\quad \lambda=2 \pi \rho \approx 100 \mathrm{~cm}$

To realize the conditions of the magnetized cooling in a non-perfectly parallel magnetic field one needs to restrict the transverse field components such as to limit the drift of the electron Larmor "circle" away from the ion during the interaction. Quantitatively, the "slow" drift angle of electrons due to the transverse field errors should not be larger than $\theta_{i}$. What solenoidal field quality does this correspond to? To answer this question, I will start with the equation of motion for an electron in the longitudinal magnetic field.

Suppose that there are transverse fields in a solenoid: $H_{x}(s), \quad H_{y}(s) \quad<<H_{0} \equiv$ const. For small transverse oscillations, the electron equation of motion can be written as:

$$
\begin{aligned}
& x^{\prime \prime}=\frac{1}{\rho} \cdot\left(y^{\prime}-\frac{H_{y}}{H_{0}}\right), \\
& y^{\prime \prime}=-\frac{1}{\rho} \cdot\left(x^{\prime}-\frac{H_{x}}{H_{0}}\right)
\end{aligned}
$$

where' is $d / d s$. After introducing new variables: $z=x+$ $i y$ and $B=H_{y}-i H_{x}$, the equation (1) can be rewritten as:

$$
\varphi^{\prime}=-\frac{i}{\rho} \cdot \varphi-\frac{B}{H_{0} \rho},
$$

where $\varphi \equiv z^{\prime}$ is a complex variable representing the electron trajectory angle in the solenoid. The solution of this defferential equation is as follows:

$$
\varphi(s)=\left[\varphi(0)-\int_{0}^{s} \frac{B\left(s^{\prime}\right) \cdot \exp \left(i \frac{s^{\prime}}{\rho}\right)}{H_{0} \rho} \cdot d s^{\prime}\right] \cdot \exp \left(-i \frac{s}{\rho}\right)
$$

It is important to note that in a solenoid a typical scale of its transverse field variation is about equal to the solenoid diameter, $D$.

The equation (3) was solved numerically (using MathCAD) for a proposed RHIC cooler parameters: $\rho=$ $16 \mathrm{~cm}, D=30 \mathrm{~cm}$. A $1-\mathrm{T}, 10-\mathrm{m}$ long, 30-cm diameter solenoid was simulated by 1001 identical current loops, placed $1 \mathrm{~cm}$ apart from $s=0$ to $10 \mathrm{~m}$. Starting from $s=$ $3.5 \mathrm{~m}$ each current loop was randomly (with a gaussian distribution) displaced transversely to create a transverse field error. The rms displacement was chosen such as to limit the field error, $H_{\perp} / H_{0}$, by a value of about $1 \times 10^{-5}$. The transverse fields were then calculated by adding transverse fields from each current loop on the solenoid axis. Figure 1 shows the result of such calculations. A number of random distributions were calculated and the results presented here are quite representative of all the runs.

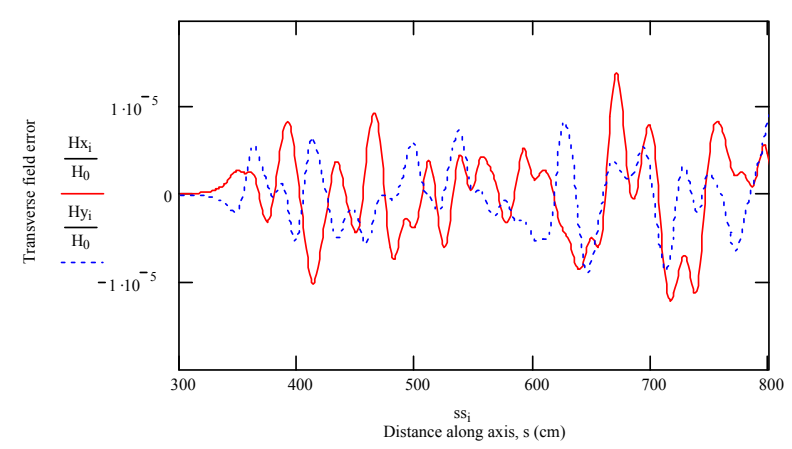

Figure 1: The horizontal and vertical field errors. The solenoid diameter is $D=30 \mathrm{~cm}$. 
Eq. (3) was integrated numerically using the above transverse field distribution and the zero initial conditions: $\varphi(0)=0$. Particles were launched at $s=300 \mathrm{~cm}$, where the transverse magnetic field is zero. The electrons were then propagated to $s=800 \mathrm{~cm}$ or for about 5 Larmor periods. This number corresponds to a number of Larmor periods in a typical ion-electron interaction. The resulting horizontal and vertical angles are presented in Fig. 2.

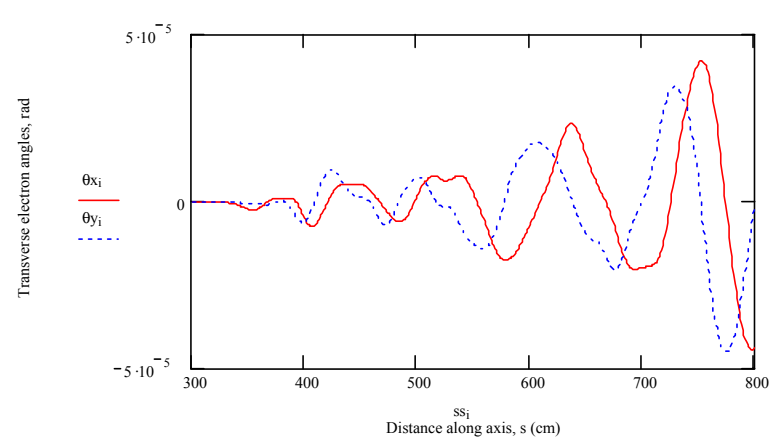

Figure 2: The horizontal and vertical angles.

The total transverse angle is increasing with distance, although from the simulations it is difficult to say if the rate is linear or the square-root of $s$, as one would expect for an oscillator, experiencing random kicks. In any case, it should be compared with thermal electron angles, which are about $6 \times 10^{-4}$. This continuous Larmor excitation can be suppressed by transverse field correctors, adjusted to minimize the integral in Eq. 3. However, if the correctors are placed over the solenoid OD, their effective length is comparable with the solenoid diameter. Such correctors are ineffective for minimizing the integral in Eq. 3.

Because of this fast Larmor excitation it is difficult to separate the Larmor motion from the drift motion. One way of doing that would be to analyze electron trajectories and to determine with what accuracy does an electron arrive to the same transverse position after each Larmor oscillation. I analyzed the electron drift angles by performing a running average of transverse angles, $\varphi(s)$, over one Larmor period. The results are presented in Figure 3.

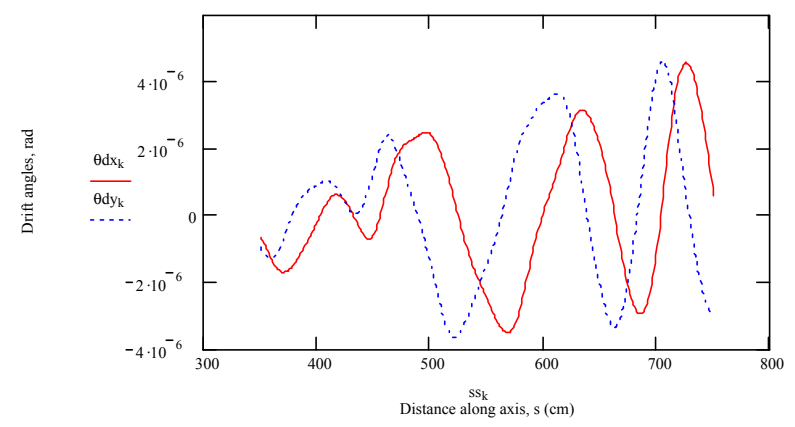

Figure 3: The horizontal and vertical drift angles, calculated by a running average of transverse angles over one Larmor period.

The proposed field quality, $H_{\perp} / H_{0} \leq \theta_{i}=1.3 \times 10^{-5}$ seems sufficient to keep drift angles under $1 \times 10^{-5}$. However, a more careful analysis of Eq. (3) for a specific solenoid design is required. It seems that by limiting the Larmor excitation of the total transverse angle it might be possible to reduce the drift angles and, thus, to relax the field quality requirements. The goal of optimization should be to bring the required field quality to about $1 \times 10^{-4}$, which is within reach of existing winding and measuring technologies.

\subsection{Low field option (Fermilab cooling system)}

The design Fermilab electron cooler and beam parameters under consideration are:

Beta-function in the cooling section: $\beta=30 \mathrm{~m}$

Pbar beam normalized rms emittance: $\varepsilon_{n}=1.6 \mu \mathrm{m}$

Pbar beam rms angular spread: $\quad \theta_{i}=7 \times 10^{-5} \mathrm{rad}$

Electron beam momentum: $\quad p c=5 \mathrm{MeV}$

Relativistic parameter: $\quad \gamma=10$

Electron beam transverse temperature: $T_{e}=0.2 \mathrm{eV}$

Electron beam rms angular spread: $\quad \theta_{e}=2 \times 10^{-5} \mathrm{rad}$

Magnetic filed in the cooling section: $H_{0}=100 \mathrm{G}$

Solenoid length:

$L=20 \mathrm{~m}$

Larmor radius (at full energy): $\quad \rho=p c / e H_{0} \approx 166 \mathrm{~cm}$

Larmor period:

Solenoid diameter:

$\lambda=2 \pi \rho \approx 10 \mathrm{~m}$

$D=15 \mathrm{~cm}$

Since $D<<\rho$, the exponent in the integrand in Eq. (3) is a slowly varying function compared to $B(s)$ and can be removed from the integral. Thus,

$$
\varphi(s) \approx \varphi(0) \cdot \exp \left(-i \frac{s}{\rho}\right)-\int_{0}^{s} \frac{B\left(s^{\prime}\right)}{H_{0} \rho} \cdot d s^{\prime}
$$

for $s<<2 \pi \rho$. The second term is the drift motion and the first term is the Larmor oscillations. Therefore, the drift angle can be limited by limiting the maximum transverse field integral along the solenoid. In the Fermilab project this maximum value of integral should be below $\pm 1 \mathrm{G} \cdot \mathrm{cm}$ [11] to keep the drift angle below $\pm 7 \cdot 10^{-5}$. It is achieved by transverse coils, which null-out the transverse field integral. Since it is the field integral which is the figure of merit and not the field itself, the restriction on the field quality is less stringent (by about a ratio of $\rho / D$ or more). Of course, long-scale field variations are more important than the short-scale ones. At Fermilab, a 4-m long prototype solenoid was manufactured, installed and measured $[12,13]$. The quality of the measured solenoid prototype was found to be satisfactory for the electron cooling purpose. Integrals of transverse fields can be made below $1 \mathrm{G} \cdot \mathrm{cm}$ at the solenoid field of $150 \mathrm{G}$, if corrector currents are at optimum. The production of a $20-\mathrm{m}$ long solenoid has began at a rate of $4-\mathrm{m} / \mathrm{month}$. It will be finished by the end of 2001 .

\section{BEAM TRANSPORT}

The proposed electron cooling system for RHIC [7] will employ an rf-bunched, $50 \mathrm{MeV}, 100 \mathrm{~mA}$ (average) electron beam. Electron cooling at Fermilab will use a DC, 4.3 MeV, 0.3 A electron beam. Both schemes require energy recovery, or recirculation, which is 


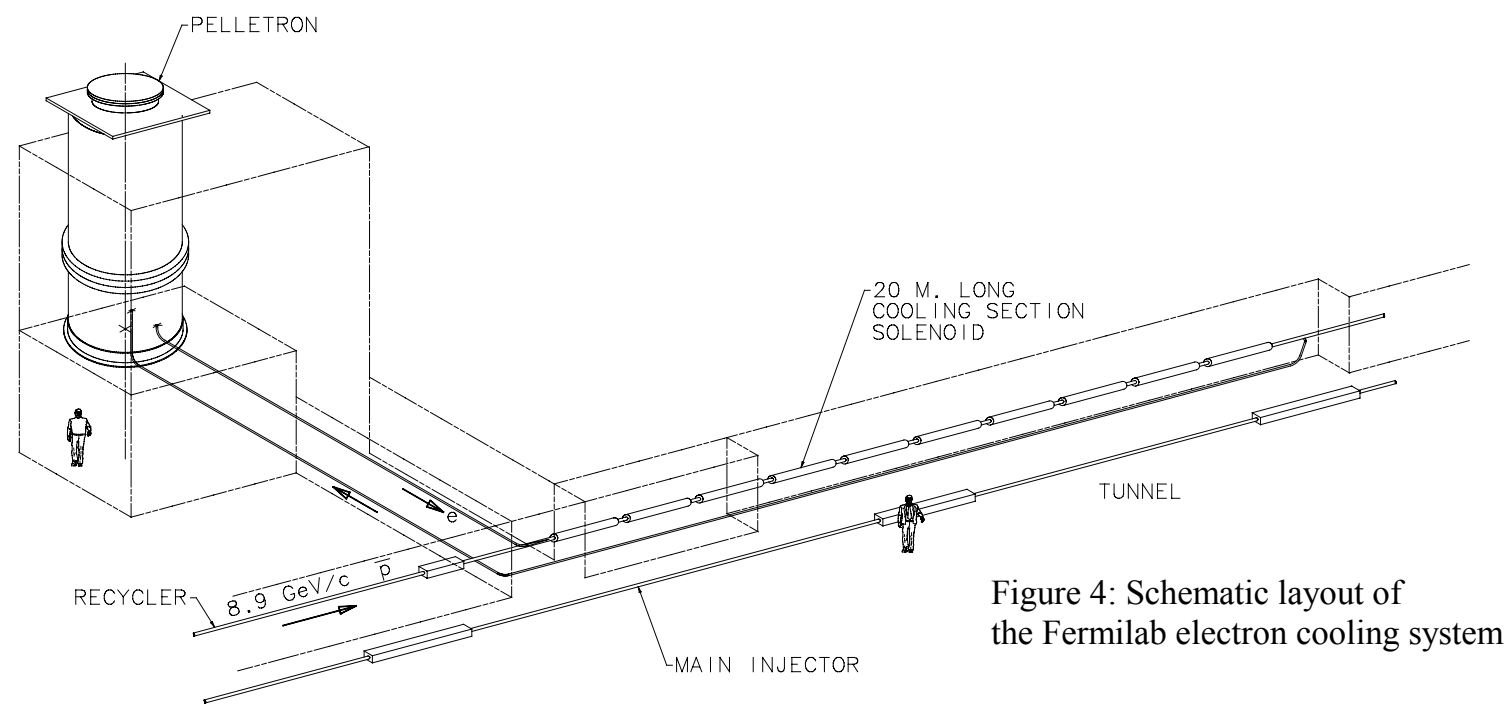

standard for all existing low-energy electron cooling devices. At Fermilab, the beam is generated in an electrostatic Pelletron[14] accelerator, then transported to the cooling section, and returned back to the high voltage terminal (Fig.4). However, the chosen transport scheme in both projects is non-standard. Only the gun (injector) and the cooling section are immersed in a longitudinal magnetic field, while a lumped focusing system is used in between [15]. Such a system can be employed if an effective beam emittance outside of the longitudinal magnetic field,

$$
\varepsilon_{e f f}=\frac{e}{2 \pi \cdot p c} \Phi,
$$

is low enough. Here $e$ is the electron charge, $c$ is the speed of light, $p$ is the momentum, and $\Phi$ is the magnetic flux at the cathode.

In any case, the maximum allowable value of $\varepsilon_{e f f}$ is dictated by the maximum allowable beam size after the beam exits the gun (injector) solenoid. At Fermilab, this maximum beam size is limited by the acceleration tube aperture. The effective emittance value at the extraction from the solenoid is about $40 \mu \mathrm{m}$ (extraction is at 1.5 $\mathrm{MeV} / \mathrm{c}$ ). In the proposed electron cooling system for RHIC this emittance is about $60 \mu \mathrm{m}$ (extraction at 2.5 $\mathrm{MeV} / \mathrm{c}$ ). The stronger the magnetic field at the cathode (for a given cathode diameter), the higher should be the electron energy where the magnetic field ends.

To form a round and cold electron beam in the cooling section, the transport line has to satisfy the following requirements [15]:

1. The magnetic flux inside the beam in the cooling section is equal to the magnetic flux at the cathode.

2. The transport map between the cathode and the cooling section is rotationally invariant.

3. Aberrations in all elements of the transport line do not increase the beam emittance significantly.

An optical channel that satisfies these requirements has been designed for the Fermilab cooler and most of the elements have been ordered. Beam tests with a full-scale beam line will begin in the spring of 2002 .

\section{ELECTRON BEAM RECIRCULATION}

An efficient electron beam recirculation [16] has always been an import issue in low energy electron coolers. The high efficiency recirculation (with relative current losses of $10^{-4}$ ) is needed at low energies mainly for two reasons: (1) to lower the energy ripple of the electron beam and (2) to reduce the beam-loss-induced gas desorption. At high energies, the beam recirculation (dc or rf-bunched) is even more important because of the required high average beam power (1 MW or more) and the strong dependence of the X-ray radiation rates on the electron beam energy. The issues of gas desorption also remains important, primarily in low-energy portions of the system.

\subsection{DC beam recirculation}

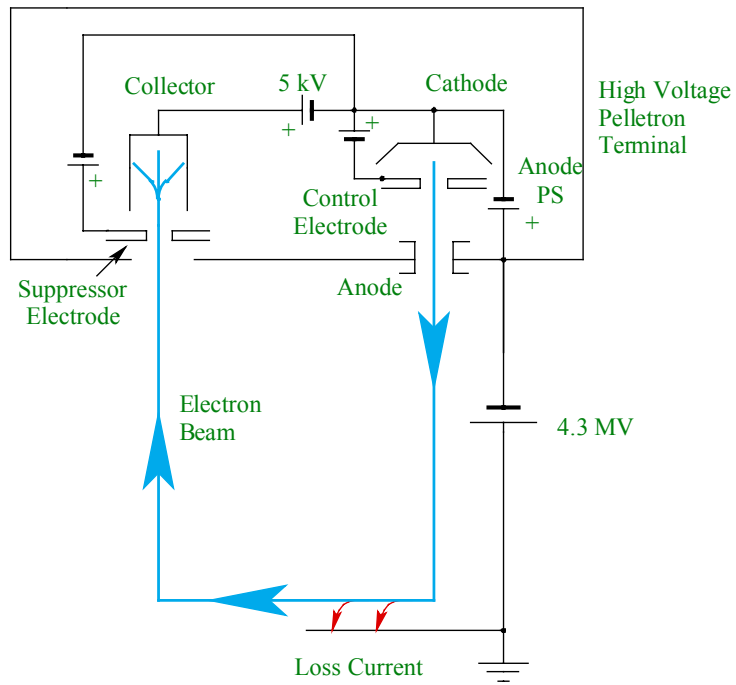

Figure 2: Simplified electrical schematic of the Fermilab recirculation system 
At Fermilab, the recirculation tests have begun on a recently commissioned 5-MV system [17]. Figure 2 shows the simplified electrical schematic of the Fermilab electron recirculation system. The goal of this recirculation test is to achieve a stable recirculation of 4.3 $\mathrm{MeV}, 0.5 \mathrm{~A}$ DC beam in a dedicated U-bend set-up, which is conceptually close to that used in the previous $1-2 \mathrm{MeV}$ range test [18]. The rate of progress in a DC beam recirculation at Fermilab is currently determined by two factors: (1) electronics damage from high-voltage sparks and (2) beam-induced outgassing of the collector surface. It is anticipated that a $0.5-\mathrm{A}$ DC electron beam current will be reached by the end of 2001 . It is also planed to test all diagnostics proposed for the full version of the cooler. In the spring of 2002 the full-scale beam line will be commissioned.

\subsection{RF-bunched beam recirculation}

For an rf-bunched electron cooling system the progress in energy-recovering linac technology has been quite rapid in a recent years, primarily due to the FEL developments. An FEL at Jefferson Lab [8] has been operated at $50 \mathrm{MeV}$ and at about $5 \mathrm{~mA}$ average beam current. It is believed that it is quite possible to beam attain currents of about $200 \mathrm{~mA}$ - suitable for the high-energy cooling system. However, there are several important differences between the FEL and the electron cooling system requirements to the electron beam. First, for the efficient electron cooling the beam cathode needs to be immersed in a longitudinal magnetic field as described in the previous section. Second, the energy spread needs to be very low (of the order of $10^{-4}$ ) and the electron bunch length in the cooling section needs to be about $50 \mathrm{~cm}$ to match a typical ion bunch length. The required repetition rate is about several MHz. All these differences grant a separate (from FEL) development program in recirculating linacs. Figure 4 shows a schematic of the proposed RHIC electron cooling system.

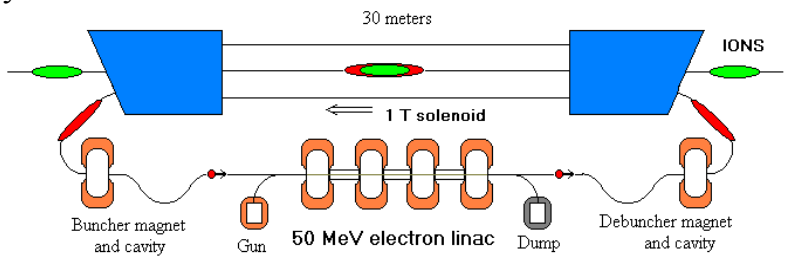

Figure 4: Schematic of a high-energy electron cooling system for RHIC [5].

\section{OUTLOOK}

To achieve an operating high energy electron cooling system many R\&D topics still need to be addressed. For the Fermilab cooler these topics are primarily related to electron beam transport, diagnostics and reproducibility. Since the Fermilab cooler design is relying upon the fact the low electron beam temperature can be preserved as the beam being delivered to the cooling section. The challenge will be to measure such low temperatures in a non-destructive manner. For a bunched-beam cooling system (such as proposed for RHIC) there are a number of questions related to the cooling process itself that need and can be addressed experimentally in a low-energy cooler. These questions are:

1. Does the bunched-beam cooling work?

2. Is the "strongly-magnetized" cooling efficient enough if the electron transverse temperatures are high (i.e. $1000 \mathrm{eV})$ ?

3. What are optimum transverse and longitudinal electron beam sizes for a given ion beam size?

\section{ACKNOWLEDGEMENTS}

I am very grateful to my colleagues and collaborators A. Shemyakin, A. Burov, Ya. Derbenev, I. Ben-Zvi, V. Parkhomchuk and V. Lebedev for allowing me to use their research results in my report.

\section{REFERENCES}

[1] G.I. Budker et al., "Experimental studies of electron cooling", Part. Accel. 7 (1976), p. 295.

[2] M. Veis et al., EPAC'98, Rome, 1988.

[3] E.I. Antokhin et al., NIM A 441 (2000), p. 87.

[4] I.N. Meshkov, "Electron cooling with a circulating electron beam in GeV energy range", NIM A 441 (2000), p. 255.

[5] K. Balewski, "Studies of electron cooling at DESY", NIM A 441 (2000), p. 274.

[6] S. Nagaitsev et al., "FNAL R\&D in medium energy electron cooling", NIM A 441 (2000), p. 241.

[7] I. Ben-Zvi, "Electron cooling for RHIC", these proceedings

[8] G.R. Neil et al., "Kilowatt lasing in a free-electron laser", EPAC 2000 (Vienna), p. 764.

[9] Ya.S. Derbenev, "Prospects of high energy electron cooling", EPAC 2000 (Vienna), p. 8.

[10] A. Skrinsky, "Continuous electron cooling for highluminosity colliders", NIM A 441 (2000), p. 286.

[11] S. Nagaitsev et al., " Cooling section solenoid for the $5 \mathrm{MeV}$ Fermilab electron cooling project", EPAC 2000 (Vienna), p.

[12] J. Leibfritz et al., "Fermilab electron cooling project: engineering aspects of cooling section", these proceedings.

[13]A. Shemyakin et al, "Fermilab Electron Cooling Project: field measurements in the cooling section solenoid ", these proceedings.

[14] Pelletron is a trademark of National Electrostatic Corporation (NEC), Middlton, WI

[15]A. Burov et al., Phys.Rev.ST -Accel.Beams,Vol.3, $094002(2000)$

[16]A. Sharapa and A. Shemyakin, in Proceedings of Beam Cooling Workshop 1993 (Montreux), CERN 94-03, p. 293.

[17]A. Shemyakin et al., "Fermilab Electron Cooling project: Commissioning of the $5 \mathrm{MeV}$ recirculation test set-up", these proceedings.

[18] A.C.Crawford et al., NIM A435 (1999), 339. 